\title{
Cardiovascular safety of COX-2 inhibition: time to inform systems of postmarketing surveillance?
}

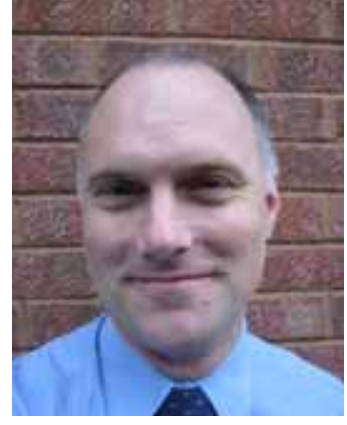

Robert J MacFadyen ${ }^{\dagger 1}$ \& Thomas M MacD onald ${ }^{2}$

${ }^{\dagger 1}$ Author for correspondence City H ospital, U niversity $D$ epartment of M edicine and $D$ epartment of Cardiology, Dudley Road, Birmingham, B18 7QH , UK

Tel.: +44 1215075080

Fax: +44 1215544083

Robert.macfadyen@swbh.nhs.uk

2U niversity of $D$ undee, $M$ edicines M onitoring U nit and $D$ epartment of Clinical Pharmacology, Division of $M$ edicine and Therapeutics, $\mathrm{N}$ inewells $\mathrm{H}$ ospital and M edical School, D undee D D 1 9SY, U K

\section{'... adverse events involving a} common disea se such ascoronary disease are harder to define than rare or idiosync ratic side effects...'

Since late summer 2004, regulatory and patient concerns over retrospective data on the overall safety of cyclooxygenase (COX)-2 inhibitors (coxibs), such as rofecoxib, valdecoxib and celecoxib, in patients with coronary disease have evolved in an ever-upward spiral of increasingly public rather than scientific debate. The episode was heralded to the wider world by the precautionary withdrawal of rofecoxib from the world market following discussions by the US Food and D rug Administration (FD A). A serious drop in the stock valuation of Merck Sharpe and Dohme Ltd (MSD) and a worldwide media frenzy followed. In particular, the US media reaction to this step was openly criticised by AJJ Wood (the Scottish clinical pharmacologist based at Vanderbilt who chaired the relevant FD A advisory panel on COX-2 inhibitors and is a potential new commissioner of the FD A).

Regulatory concern over data on safety is not a new feature in the assessment of drugs following marketing. Paradoxically, adverse events involving a common disease such as coronary disease are harder to define than rare or idiosyncratic side effects. Unlike the latter, they tend to be below the limits of recognition of individual clinicians and most current systems of postmarketing surveillance. Specifically, recognition of an impact of concomitant drug treatment on coronary disease in patients with arthritides (where the background symptom burden is traditionally very high) is a good case study in the complexity of pharmacoepidemiology. These patients have a long-established excess incidence of coronary and vascular events recognized long before the era of COX-2 inhibitor use [1]. The safety and efficacy of any drug entity (new or old) is of course a product of the number and duration of patient exposures and the unpredictable impact of prescription to unselected patients (those not systematically studied during development). C learly there were no data to suggest an adverse interaction with coronary disease or its treatment before marketing. In general, no drug therapy is anything other than relatively safe in this regard and, routinely, prescribers place a disproportionate amount of faith in the developmental and regulatory assessment process. W hat can be learnt from the regulatory process laid out in the postmarketing surveillance of cox-2 inhibition?

\section{COX-2 inhibitor treatment \& coronary patients in context}

The facts are clear. The class of COX-2 inhibitors has had a large global postmarketing patient exposure, predominantly in pain control, where they are effective medicines. W ithin this experience, a variety of initially noncardiologic endpoint studies (most with gastrointestinal side effect end points, but some concerned with ancillary beneficial targets such as on bowel polyps) found a modest absolute rise in repeat coronary events (myocardial infarction and stroke) in patients with a known coronary history who received a COX-2 inhibitor. The relative nature of the impact (a mean twofold relative risk of stroke and coronary events) is in the context of a low absolute rate of coronary events in the region of 0.1 versus $0.4 \%$ in the VIoxx G astrointestinal O utcome Research (VIGO R) study for naproxen versus rofecoxib (in 8076 patients with rheumatoid disease) where the comparator naproxen may have had some cardioprotective, antiplatelet effect (exhibited via COX-1) [2]; and no adverse effect at all in the Celecoxib Long term Arthritis Safety Study (CLASS) trial of celecoxib versus ibuprofen or diclofenac in 7968 patients with osteoarthritis (OA) or rheumatoid disease [3]. In a subsequent large, retrospective analysis of 378,776 patients from the Tennessee M edicaid program, Ray and colleagues suggested a risk ratio of 1.70 for rofecoxib and 1.78 for celecoxib, compared with nonusers, but they could not exclude a disease-associated risk [4]. Similar retrospective studies of rofecoxib gave a similar relative risk (1.69) in 28,000 rofecoxib users compared with naproxen. Finally, we had a small-scal e study of parecoxib or valdecoxib use in 469 patients undergoing coronary artery surgery, which 
showed a 15 versus $9 \%$ incidence of adverse effects, but these were mostly of wound infection (10 vs. 0) and a nonsignificant increase in cerebrovascular complications, myocardial infarction and renal dysfunction, which were proportionally greater, but not significantly different, between the groups [5]. This is set against the well publicized primary benefits that these drugs provide to millions of individual patients, including coronary-surgery patients [5], generally after therapeutic failures with an alternative nonsteroidal anti-inflammatory drug (N SAID). Given the aforementioned concerns over interactions between aspirin and NSAIDS[6] and recent events over the use of a range of COX-2 inhibitors in coronary disease [7], can we monitor them better after release?

\section{Prescribing decisions for \\ individual patients}

We know that the factors that initiate prescription of a given drug in routine use are complex, even within an intended target population [8]. Extending from this, the interaction of drugs with disease in patients with multiple diseases, given multiple concomitant drug treatments, is a constant source of unpredictable and unpredicted adverse events. There are no current reliable prospective data set to define this process in any setting. While regulators in general have been condemned in rather shrill tones by some [9] (perhaps with good reason, for focusing too keenly on definition of efficacy), in reality, the events surrounding COX-2 inhibition more than illustrate the fact that we are making no progress in broadening the system of surveillance, while at the same time facilitating safe and cost-effective drug development and assessment. Even after the recent decisions over the use of COX-2 inhibitors, we cannot expect all drugs to be tested in all patient groups before regulatory approval.

In the real world (away from clinical or regulatory trials), both new and old drugs tend to be used (and in some eyes at least, abused) in different ways from those that either the interested spe cialist, manufacturer or regulatory authorities had ever thought relevant. Regulatory approaches based on exclusive and controlled use realistically (and possibly correctly) still have little practical impact on the treatment for individual patients. From the standpoint of the concerned prescriber responding to an individual patient, there always seems to be a case to treat the patient, regardless of warnings and restrictions. There may be the case that persuasive direct or indirect advertising or promotion can overcome reluctance to prescribe within the restricted boundaries of regulatory approval. Frankly, some interactions are misrepresented by small studies or incorrect logic that drugs may be beneficial, where this is clearly not the case on broader analysis. There is evidence that this may be the case for COX-2 inhibitors, where some small studies using surrogate end points suggested beneficial effects in cardiac patients where the detailed pharmacology of the class suggested quite the opposite.

'...the factors that initiate presc ription of a given drug in routine use are complex, even within an intended target population...'

The emergence and recognition of unpre dicted or unstudied adverse events takes a considerable amount of time, in part, due to the inefficient process of documentation, but more exactly due to the fact that patient experiences (and this might most logically be cut off at those experiences that require hospitalization) cannot be linked to their drug treatment in real time. Real-time monitoring of efficacy (the balance of adverse events and benefit) and cost efficacy is poor. O nly rarely are drugs given regulatory approval with any meaningful commitment to monitoring adverse events.

Once again, we see major editorial debate expose the unreal expectation that drugs (in general, and COX-2 inhibitors in particular) are 'not safe' [10]. This tends to suggest lack of insight that any exogenous material cannot be safe all the time and in every patient, given that even a placebo treatment has easily recognized adverse effects.

Adverse drug interactions with coronary disease are particularly important given the prevalence of this disease on a global scale. Nonetheless, the extent of drug-disease interaction testing is limited where the agent in question might not obviously be a candidate for a primary interaction with either the control systems or direct physiology or pathology of the heart. Is it the case that COX-2 inhibitors would be so regarded? W ith the fundamental role of COX inhibition in antiplatelet therapy, one would have thought not, even based on a superficial analysis.

While many development programs of drugs not primarily intended for cardiovascular use, often employ an analysis of safety, this is 
frequently superficial. This is not to suggest that there are not large numbers of cardiovascular specialists eager to examine the cardiovascular effects of noncardiac drugs. This was certainly the case with COX-2 inhibitors, whose profile was highlighted by selective subanalyses of safety data early after marketing.

'...the extent of the drug-disease

interaction testing is limited, where

the agent in question might not

obviously be a candidate for a

primary interaction...'

The routine testing of noncardiac drugs is often superficial. For example, effects on the surface electrocardiogram (ECG) of normal volunteers are frequently completed as a part of Phase I trials for impact on rhythm or ECG intervals. $H$ owever the surface ECG is such a nonsensitive and nonspecific tool even in patients with known coronary disease the value of this exercise in predicting patient response is patently limited.

In order to continue to support and develop new and successful treatments, the pharmaceutical industry should be guided by the medical community, patients and regulators to underline safety and better define the inevitable profile of adverse events intergral to the use of any drug treatment.

Could the adverse cardiac effects have been predicted?

Certainly M ukherjee, $\mathrm{N}$ issen and Topol raised the issue indirectly from reanalysis of trials, data or database material based on assessing the efficacy and gastrointestinal tolerance of two COX-2 inhibitors (celecoxib and rofecoxib) against placebo [7]. As it turns out, an active control might have been more relevant; but importantly, the authors made it clear that randomized studies specifically powered and set up to carefully define cardiovascular safety were required to answer what at that stage seemed to be something worth clarifying for confounding effects. The irony is that new data in this structured form specifically addresssing safety has not appeared. What has generated the concern is simply more ad hoc reanalysis. These authors would be the first to admit that the number of events recorded here was small and the follow up short. Confusingly, in assessing this data, these authors were in fact more interested in the impact of concomitant aspirin for which they could find no interaction. Subsequent small studies have understandably explored the impact of COX-2 inhibitors as beneficial agents for coronary disease.

Can large scale active monitoring of drug safety (and/or efficacy) work at a practical level?

The current system of passive adverse event reporting has little impact in defining new adverse event risks, although they can serve as an after-the-event system of confirmation or documentation. What is required is proactive monitoring of patterns of clinical events and hospitalization, while minor interactions not requiring hospital admission will still occur. It is time that regulators and the industry joined together to address this nationally and internationally. It needs the support of patients and, by definition, their general practitioners and governments to buy into a real-time system of major common clinical-event monitoring to provide adverse-event linkage to drug therapy. $\mathrm{N}$ aturally, this should be based around major cardiac and vascular event rates (as well as possibly common cancers?) as these dominate morbidity and mortality and are easily recognizable at the point of hospital admissions. It is the complexity of the human rights issues involved here (much more than the digital records linkage that already exists in limited centers) to allow real-time event monitoring at a population level. These should not be seen as insurmountable, rather the subject of public debate and support.

\section{Denominators}

In every sampling exercise, the critical factor defining the sensitivity of an estimate is the scope and size of the sample. It is logical that adverse events will happen and the task is to try to anticipate and define the risk of serious events in specific groups where the injury is either serious or avoidable. It was not possible to suspect that COX-2 inhibitors would have had a significantly worse profile than NSAIDs, which had been in use for decades in coronary patients in one form or another.

\section{Mechanisms}

It is only reasonable to suspect and prospectively monitor activity and safety if there is a reasonable link through a known mechanism of action between a drug class and a potentially vulnerable patient group.

\section{Money}

While we do see some cases of prospective monitoring of drugs at present, it is uncommon. 
Equally, there would be little argument that restricted application of such a monitoring expense would be a disincentive to development and a restriction to widespread use. However, automated systems to monitor common adverse events through records linkage (and applicable to all drugs) need not be prohibitively expensive and might be incorporated into the accounting for new drug budgets across a wide range of new agents rather than seen as specific issues for one or two drugs. Money is mostly required to finance the setting up of large-scale systems in a range of countries, which accomodate pharmacogenetic diversity as well as differing patterns of use and can then serve as a global database. Continuing a system of anonymized digital records, linkage for major clinical end points to analyze the relationship to multiple drug exposure in large numbers of admissions is then both practically and financially feasible.

\section{Legality \& right to privacy}

Clearly, if patients are to benefit from treatment, they may also be willing to accept that their anonymous clinical experience is valuable in the monitoring and tailoring of drug therapy.

In the end, a proactive system of drug-event linkage need not give rise for concern to the industry. The costs can be incorporated on a global scale to provide a large database in one or more markets. It would involve all the best aspects of partnership between patients, their physicians, regulators, government and most importantly, the industry. They all share the common goal of safe, effective and informed medicines use. Problems need to be put in context and understood without ill-informed over reaction to what is an undeniable truth - all drug treatments have a balance of beneficial and adverse effects.

The future does not necessarily need to be more of the same heated debate filled with emotional reactions and superficial legal challanges. Proactive monitoring should be put in place in order to assess this risk and inform as to whether it is acceptable or unacceptable not to question its existence, which should be assumed. Cardiovascular event analysis, given the sheer presence and significance of this pathology, should be a priority. Retrospective ad hoc or meta-analyses are valid techniques in order to explore a hypothesis (much as originally published by M ukherjee and colleagues) but cannot be a preferred option. It is ineffective, cumbersome and can lead to both false-positive and -negative relationships. We require national or international case-record linkage funded by regulatory authorities for adverse drug events (both for new and old agents) that allow us to use goodquality data to address both within- and between-class cardiovascular risks.
Bibliography

1. M onson RR, H all AP. M ortality among arthritics. J. Chron. Dis. 29, 459-467 (1976).

2. Bombardier C, Laine L, Reicin A. Comparison of upper gastrointestinal toxicity of rofecoxib and naproxen in patients with rheumatoid arthritis. N . Engl. J. M ed. 343, 1520-1528 (2000).

3. Silverstein FE, Faich $\mathrm{G}$, G oldstein JL. Gastrointestinal toxicity with celecoxib vs nonsteroidal anti-inflammatory drugs for osteoarthritis and rheumatoid arthritis: the CLASS study: a randomized controlled trial. C elecoxib Long-term Arthritis Safety Study. JAM A 284, 1247-1255 (2000).

4. Ray WA, Stein CM , D augherty JR, H all K, Arbogast PG, Griffin M R. COX-2 selective nonsteroidal anti inflammatory drugs and risk of serious coronary heart disease. Lancet 360 , 1071-1073 (2002).

5. Ott E, N ussmeir N A, D uke PC et al. Efficacy and safety of the cyclooxygenase 2 inhibitors parecoxib and valdecoxib in patients undergoing coronary bypass surgery. J. Thor. Cardiovasc. Surg. 125, 1481-1492 (2003).

6. Garcia Rodriguez $L$, Varas $C$, Patrono $C$. $D$ ifferential effects of aspirin and non aspirin nonsteroidal anti-inflammatory drugs in the primary prevention of myocardial infarction in post menopausal women. Epidemiology 11, 382-387 (2000).

7. Mukherjee D, Nissen SE, Topol EJ. Risk of cardiovascular events associated with selective C OX-2 inhibitors. JAM A 286, 954-959 (2001).

8. M acD onald TM, Pettitt $D$, Lee FH, Schwartz JS. Channelling of patients taking N SAID s or cyclooxygenase 2 specific inhibitors and its effect in relation to interpretation of outcomes. Rheumatology 42(Supp. III), S3-S10 (2003).

9. No Authors Listed. Vioxx: lessons for $\mathrm{H}$ ealth C anada and the FD A. Can. M ed. Assoc. J. 172(1), 5 (2005).
10. Finckh A, Aronson M D. Cardiovascular risks of cyclooxygenase-2 inhibitors: where we stand now. Annu. Intern. M ed. 142, 1-3 (2005).

Affiliations

Robert] M acFadyen, BSC, M D , PhD , M RPharmS, FRCPE, Consultant Cardiologist and Senior Lecturer in M edicine, City H ospital, U niversity D epartment of $M$ edicine and $D$ epartment of Cardiology, D udley Road, Birmingham, B18 7Q H, UK

Tel.: +44 1215075080

Fax: +44 1215544083

Robert.macfadyen@swbh.nhs.uk

Thomas M M acD onald, BSC, M D, FRCPE, FFPM , Consultant Physician and Professor of Pharmacoepidemiology, U niversity of D undee $M$ edicines $M$ onitoring U nit and $D$ epartment of Clinical Pharmacology, D ivision of M edicine and Therapeutics, $\mathrm{N}$ inewells $\mathrm{H}$ ospital and M edical School, D undee D D 1 9SY, U K 